\title{
THE JUNE MEETING IN PORTLAND
}

The five hundred fourth meeting of the American Mathematical Society was held at Reed College, Portland, Oregon, on Saturday, June 19, 1954, following the meeting on Friday of the Pacific Northwest Section of the Mathematical Association of America.

Attendance at the meetings was approximately 85, including 63 members of the Society.

By invitation of the Committee to Select Hour Speakers for Far Western Sectional Meetings, Professor V. L. Klee, Jr., of the University of Washington delivered an hour address entitled Convex sets in linear spaces. Professor Klee was introduced by Professor R. A. Beaumont.

There were two sessions for contributed papers, at which Professors A. T. Lonseth and Harold Chatland presided.

On Friday evening preceding the meeting there was a joint dinner of the Society and Association, at which the visitors were greeted by President Ballantine of Reed College.

Abstracts of papers presented at the meeting follow. Abstracts whose titles are followed by " $t$ " were presented by title. Mrs. Lehmer was introduced by the Associate Secretary, Mr. Rall by Professor Lonseth, Professor Nash by Professor W. T. Martin, and Mr. Krabble by Professor C. B. Morrey.

\section{Algebra AND Theory of Numbers}

\section{8t. W. E. Barnes: Primal ideals in noncommutative rings.}

In any associative ring $R$ an element $x$ is not right prime (nrp) to an ideal $A$ if $y R x \subseteq A$ for some $y \notin A$. An ideal is primal if the elements nrp to it form an ideal. These definitions differ from those of Curtis (Amer. J. Math. vol. 76 (1952) pp. 687700) but reduce to them for rings with unit and A.C.C. for ideals. They also reduce to Fuchs' (Proc. Amer. Math. Soc. vol. 1 (1950) pp. 1-8) for commutative rings. An ideal $B$ is nrp to $A$ if every element of $B$ is nrp to $A$. Maximal nrp to $A$ ideals always exist and their intersection is called the adjoint of $A$. In a class of rings, called uniform, the maximal nrp ideals of any ideal are prime. The A.C.C. implies uniformity, but not conversely. Results (similar to the classical Noether theory) on representations of an ideal as the intersection of primal ideals with prime adjoints are obtained which include those of Fuchs and Curtis. (Received May 3, 1954.)

589. W. E. Barnes: Principal component ideals in noncommutative rings.

If $A$ and $B$ are ideals in any associative ring such that $A \subseteq B$, the lower right isolated $B$-component of $A, L(A, B)$, is the ideal sum of all ideals $A m^{-1}$, where $m$ is right prime to $B$ (see previous abstract) and $A m^{-1}=\{x \mid x R m \subseteq A\}$. The upper right isolated $B$-component of $A, U(A, B)$ (which always contains $L(A, B)$ ) is the intersection 
of all ideals $C \supseteq A$ and such that every $m$ right prime to $B$ is right prime to $C$. If $B$ is a maximal nrp to $A$ ideal, then $L(A, B)$ and $U(A, B)$ are called lower and upper right principal components of $A$. For $B$ a prime ideal in a commutative ring, these definitions reduce to those of W. Krull. It is shown that every ideal in an associative ring is the intersection of its lower right principal components, and under certain conditions is also the intersection of its upper right principal components. (Received May 3, 1954.)

\section{0t. H. W. Becker: Latin orthotopes. Preliminary report.}

These are rectangular figures in space of any number of dimensions, whose line elements are unrepetitive permutations in any direction parallel to the axes. $L_{i ; f}^{m ; n}$ is the number of $L O$ with $n$ finite axes of $f$ letters, and $m$ axes of $i$ letters, $i \rightarrow \infty . W_{i ; f}^{m: n}$ $=i^{m_{f}{ }^{n}}$ is the corresponding number of word orthotopes, that is, $m+n$ dimensional crosswords $=$ harmonies $=$ permutations repetitive or not. $W \supset L$, and $L_{i: n}^{m: n}$ $\sim(N / D) i^{i_{f} f^{n}}$, where $N=N(\pi, i, m, f, n), D=D(e, i, m, f, n)$, generalizing $L_{i: f}^{i: 1}$ $\sim(i !)^{f} / e^{f(f-1) / 2}$, Amer. Math. Monthly vol. 50 (1943) p. 512; Erdös and Kaplansky, Amer. J. Math. vol. 68 (1946) p. 230. For $f=i g / h$, a correction gives $L_{i: i g / h}^{1 ; 1}$ $\sim N\left(i / e^{3 / 2}\right)^{i^{2} g / h} ; L_{i}^{2} \sim(i !)^{i} / e^{i(i+1) / 2}$. This approximation for $L_{7}^{2}$ is almost perfect, by Norton's enumeration as corrected by Sade (Marseilles), Ann. Math. Statist. vol. 22 (1951) p. 306, Mann and Sade, Math. Reviews vol. 10 (1949) p. 278, verified by Yamamoto (Fukuoka), Bull. of Math. Stat. vol. 5 (1952) p. 1; but for $L_{8}^{2}$ it exceeds the estimates of Norton and Sade. Since $L_{i}^{m} \sim N\left(i / e^{(m+1) / 2}\right)^{i^{m}}$, the chance of $m$-dimensional derangement is $L_{i: 2}^{m ; 1} /\left(L_{i}^{m}\right)^{2} \sim 1 / e^{i^{m-1}}$. These results grew out of contacts with D. H. Lehmer and H. S. M. Coxeter (who coined the title, generalizing p. 192, Math. Recreations and Essays, 1940), Amer. Math. Monthly vol. 51 (1944) p. 307; see Riordan, ibid. vol. 49 (1952) p. 160. Like swinging 3 bats before stepping up to the plate, they make Latin $\$$ seem easier by comparison; as a punching bag, they may serve to sharpen others' attack on the moot $N / D$. (Received April 22,1954.)

\section{1t. H. W. Becker: New Diophantine double and triple systems.}

The system $x^{2}+y^{2}=u^{2}, u^{2}+y^{2}=\square$ is impossible (R. D. Carmichael, Diophantine analysis, p. 14). But $x^{2}+y^{2}+z^{2}=t^{2}, t^{2}+y^{2}=q^{2}$ has the solution $q, t=\left(6 r^{2}+s^{2}\right)^{2} \pm(2 r s)^{2}$, $x=36 r^{4}-s^{4}, z=(2 r s)^{2}, y=4 r s\left(6 r^{2}+s^{2}\right)$. General solution depends on that of $R^{4}-6 R^{2} S^{2}$ $+S^{4}=2[s]=q^{2}-t^{2}$, an Eels quadrilateral (EQ) with long odd side $=\square$ and both Pythagorean $\$$ primitive. A solution of $x^{2}+y^{2}+z^{2}=t^{2}, t^{2}+y^{2}=\square, x^{2}-z^{2}=\square$ is to choose $2 r s=\square$ in $t, x=9 r^{2} \pm 4 r s+s^{2} ; y, z=2(3 r \pm s)(2 r s)^{1 / 2}$. Then $\left(t^{4}-y^{4}\right)\left(x^{4}-z^{4}\right)=\square$, discriminant of a Petrus vector, here the Desboves-Lenhart. The smallest example of the kind is $t, y, x, z=21,20,5,4 ; t, y$ and $x, z$ are also the Pythagorean parameters of an EQ with both odd sides $\Omega$ and the component $\$$ both primitive, the smallest example being $9^{2}+40^{2}=41^{2}, 41^{2}+840^{2}=841^{2}$. An explicit solution of such $X^{2}+Y^{2}$ $=U^{2}, U^{2}+Z^{2}=T^{2}$ is $T, X=\left(36 r^{4} \pm 16 r^{2} s^{2}+s^{4}\right)^{2} ; Y, Z=8 r^{2} s^{2}\left(6 r^{2} \pm s^{2}\right)\left(36 r^{4} \pm 8 r^{2} s^{2}+s^{4}\right)$; $U=1296 r^{8}-56 r^{4} s^{4}+s^{8}$ : whence cyclic quads. with 2 sides [s], as $1681,9 \cdot 841$, $41 \cdot 840,40 \cdot 841$, etc. A solution of Euler's problem $\epsilon^{2}\left(\zeta^{2}-\theta^{2}\right), \epsilon^{4}-\zeta^{2} \theta^{2}=[\$$ (Bull. Amer. Math. Soc. vol. 60 (1954) p. 136) is $[\epsilon, \zeta, \theta]=\left[(T X)^{1 / 2}, U, T\right.$ or $\left.X\right]$ $=\left[\pi\left(9 r^{4} \pm 16 r^{2} s^{2}+4 s^{4}\right), 81 r^{8}-56 r^{4} s^{4}+16 s^{8},\left(9 r^{4} \pm 16 r^{2} s^{2}+4 s^{4}\right)^{2}\right]$, in terms of the other solution to the above EQ. Only tabular solutions of $t^{2} \pm\left(y^{2}+z^{2}\right)=\square$ are known. Look up a congruent number $=2[S]$ (Dickson's History II, Chap. XVI), e.g., the first one, 5. Then $\left(5^{2}+4^{2}\right)^{2} \pm 4 \cdot 5 \cdot 4\left(5^{2}-4^{2}\right)=41^{2} \pm\left(12^{2}+24^{2}\right)=49^{2}, 31^{2}$. Can any pair of these $t, y, z$ etc. be sides of a Pythagorean $\triangle$ ? (Received April 22, 1954.) 
592t. H. W. Becker:Parametric solutions of $(\mathrm{I})\left(A^{4}-B^{4}\right)\left(C^{4}-D^{4}\right)=\square$.

None but numerical solutions are given in Dickson's History II explicitly: Euler, p. 448; Gerardin, p. 458. If $(a, b ; c, d)=a b c d\left(a^{2}-b^{2}\right)\left(c^{2}-d^{2}\right)=\square$, its 2nd Petrus transform is $\left(\left(a^{2}+b^{2}\right)^{2}, 4 a b\left(a^{2}-b^{2}\right) ;\left(c^{2}+d^{2}\right)^{2}, 4 c d\left(c^{2}-d^{2}\right)\right)$, from which a solution of (I) is $\{A, B ; C, D\}=\left\{a^{2} \pm 2 a b-b^{2} ; c^{2} \pm 2 c d-d^{2}\right\}$. Substituting Euler's forms of p. 668 ibid., one gets $\left\{r, s ; r\left(r^{8}+6 r^{4} s^{4}-3 s^{8}\right), s\left(3 r^{8}-6 r^{4} s^{4}-s^{8}\right)\right\}$; Rolle's, $\left\{r^{2} \pm 2 r s-s^{2}\right.$; $r^{8}+s^{8} \pm 8 r s\left(r^{6}-s^{6}\right)-12 r^{2} s^{2}\left(r^{4}+s^{4}\left( \pm 8 r^{3} s^{3}\left(r^{2}-s^{2}\right)+38 r^{4} s^{4}\right\}\right.$; Euler-Hillyer's, $A, D, B, C$ $=9 r^{4}+4 r^{2} s^{2} \mp 4 r s^{3}-s^{4}, 9 r^{4} \mp 12 r^{3} s-4 r^{2} s^{2}-s^{4}$. Or, specialize Petrus vectors, to get: Euler-Fermat II, $\left\{27 r^{4}+s^{4}, 4 r s^{3} ; 3 r^{4}+s^{4}, 4 r^{3} s\right\}$; Rolle, $\left\{r, s ; r^{4} \pm 2 r^{2} s^{2}-s^{4}\right\}$; DesbovesLenhart, $A, C, B, D=9 r^{4} \pm 8 r^{2} s^{2}+4 s^{4}, 4 r s\left(3 r^{2} \pm 2 s^{2}\right)$. The two smallest numerical solutions, Euler's $\{3,2 ; 9,7\}$ and $\{3,2 ; 11,2\}$ are D-L and E-H P.V., but mavericks as to (I). Also mavericks as yet are their triad $\{9,7 ; 11,2\}$, and $\{7,3 ; 17,1\}$, $\{14,5 ; 18,1\}$, and $\{17,9 ; 29,11\}$ of Gerardin's table. From Gerardin's forms, ibid. p. 647, a Pythagorean tetrahedron is deduced, whose 1st Petrus transform gives the solution of (I): $\left\{r^{6} \pm 3 r^{5} s-2 r^{4} s^{2}+r^{2} s^{4}+s^{6} ; r^{6}+r^{4} s^{2}-2 r^{2} s^{4} \pm 3 r s^{5}+s^{6}\right\}$. If $i j k l,\left(i^{2}+j^{2}\right)$ $\left(k^{2}+l^{2}\right)=[\mathrm{S}$, the 2nd Petrus transform of this Pyth. tet. is the solution of (I) $\{i \pm j ; k \pm l\}$, e.g.: the Rignaux $\left\{r^{8} \pm 6 r^{6} s^{2}-5 r^{4} s^{4} \pm 12 r^{2} s^{6}+4 s^{8} ; r^{8}-8 r^{4} s^{4}-2 s^{8}, r^{8}\right.$ $\left.+4 r^{4} s^{4}-14 s^{8}\right\} ;$ D-L, $A, C, B, D=7 r^{4} \pm 16 r^{3} s+54 r^{2} s^{2} \pm 32 r s^{3}-s^{4}, r^{4} \mp 32 r^{3} s-54 r^{2} s^{2}$ F $16 r s^{3}-7 s^{4}$; D-L, $\left\{729 r^{4}-54 r^{2} s^{2} \pm 16 r s^{3}+s^{4} ; 9 r^{4} \pm 16 r^{3} s-6 r^{2} s^{2}+s^{4}\right\}$; transformed D-L, $A, D, B, C=243 r^{5} \pm 243 r^{4} s-6 r^{3} s^{2} \mp 14 r^{2} s^{3}+7 r s^{4} \mp s^{5}, 243 r^{5} \pm 189 r^{4} s+42 r^{3} s^{2} \mp 2 r^{2} s^{3}$ $-9 r s^{4} \pm s^{5}$. (Received May 3, 1954.)

593t. H. W. Becker: The forms of E. T. Bell for modified Martin trefoils and Pythagorean tetrahedrons.

A. Martin sought Pythagorean whose 3 sides are hyps. of other Pyth. \$. In Scripta Mathematica, J. Ginsburg (June 1945) found an infinity of solutions, and E. T. Bell (March 1946) the general solution. Bell's forms easily modify, so 1, 2, or 3 of the sides are legs instead of hyps. of other Pyth. $\$$. If 1 or 3 , and corresponding pairs of sides are equal, the trefoil folds into a Pyth. tet.: $U^{2}+Z^{2}=V^{2}+X^{2}=T^{2}$, $V^{2}-Z^{2}=U^{2}-X^{2}=Y^{2}$. If $Y$ is more even than $Z$, the forms are those of Bell after the changes: $a_{1}^{2}+b_{1}^{2} \leftrightarrow a_{1}^{2}-b_{1}^{2} ; p, q, r, s, t, u, v, w, x \rightarrow U, Y^{\prime}, X, Y, Z, V, U^{\prime}, Z^{\prime}, T ; Y=Y^{\prime}$, etc. If $Y$ is less even than $Z$, additionally $2 a_{2} b_{2} \leftrightarrow a_{2}^{2}-b_{2}^{2}$. If $Y$ is odd, instead $2 a_{1} b_{1}$ $\leftrightarrow a_{1}^{2}+b_{1}^{2}, 2 a_{4} b_{4} \leftrightarrow a_{4}^{2}-b_{4}^{2}, U \leftrightarrow Y^{\prime}$. The $a_{n}, b_{n}$ are the sq. roots of the sq. factors of $(T \pm U) / 2$, etc. There are 4 transforms $E ; F ; G ; H$ under which $U, V, X, Y, Z, T$ $\rightarrow T Y, V X, V Y, X Y, X Z, U V ; U Z, T Y, X Z, Y Z, U Y, U V ; U V, T Z, T Y, X Z, V Z$, $T V ; T X, U V, U X, X Z, T Y, T U$. Then there are 5 pairs of $a_{n}, b_{n}$, a different 4 of which are used in each of the Pyth. tets. $I, E I, F I, G I, H I$. The Euler-Hillyer $a_{n}, b_{n}$ $=3 r^{2}+s^{2}, 2 r s ;(3 r \mp s)(r \pm s) ; 9 r^{4}-s^{4}, 8 r^{2} s^{2} ; 9 r^{4} \mp 2 r^{2} s^{2}+s^{4}, 2 r s\left(3 r^{2} \pm s^{2}\right)$. The Desboves-Lenhart $a_{n}, b_{n}=\left(3 r^{2}+6 r s+2 s^{2}\right)\left(9 r^{2}-6 r s-2 s^{2}\right), 4 r^{2}(3 r+2 s)^{2} ;\left(3 r^{2}+2 r s-2 s^{2}\right)$ - $\left(21 r^{2}+14 r s+2 s^{2}\right), 2 r(3 r+2 s)\left(3 r^{2}+2 r s+2 s^{2}\right) ; \quad\left(15 r^{2}+10 r s+2 s^{2}\right)\left(3 r^{2}+2 r s+2 s^{2}\right)$, $8 r s(3 r+s)(3 r+2 s) ; 9 r^{2}+6 r s+2 s^{2}, 4(3 r+2 s) ; \quad\left(21 r^{2}+14 r s+2 s^{2}\right)\left(3 r^{2}+2 r s-2 s^{2}\right), 9 r^{4}$ $+12 r^{3} s+40 r^{2} s^{2}+24 r s^{3}+4 s^{4}$. The Rolle $a_{n}, b_{n}=4 r s\left(r^{2}+s^{2}\right), r^{4}-6 r^{2} s^{2}+s^{4} ; 2\left(r^{4}-s^{4}\right)$, $r^{4}-6 r^{2} s^{2}+s^{4} ; r\left(r^{4}+6 r^{2} s^{2}-3 s^{4}\right), s\left(3 r^{4}-6 r^{2} s^{2}-s^{4}\right) ; r\left(r^{4}-2 r^{2} s^{2}+5 s^{4}\right), s\left(5 r^{4}-2 r^{2} s^{2}+s^{4}\right)$; $r^{8}+20 r^{6} s^{2}-26 r^{4} s^{4}+20 r^{2} s^{6}+s^{8}, 2\left(r^{2}+s^{2}\right)^{2}\left(r^{4}-6 r^{2} s^{2}+s^{4}\right)$. (Received May 3, 1954.)

594t. R. L. Blair: $A$ note on f-regularity in rings.

An element $a$ of a ring $A$ is f-regular (cf. Trans. Amer. Math. Soc. vol. 75 (1953) pp. 136-153) in case $a \in(a)^{2}$, where (a) denotes the ideal of $A$ generated by $a$. An 
ideal of $A$ is $f$-regular in case each of its elements is $f$-regular. The existence of a largest $f$-regular ideal $F(A)$ of $A$ and the fundamental relation $F(A / F(A))=0$ follow from general considerations of Brown and McCoy (Trans. Amer. Math. Soc. vol. 69 (1950) pp. 302-311). The list of "radical-like" properties of $F(A)$ is rounded out by showing that if $B$ is an ideal of $A$ then $F(B)=B \cap F(A)$ and that, if $A_{n}$ denotes the complete matrix ring of order $n$ over $A$, then $F\left(A_{n}\right)=(F(A))_{n}$. The location of $F(A)$ with respect to the Jacobson radical $J(A)$ and the prime radical $N(A)$ of McCoy is considered. It is shown that $N(A) \cap F(A)=0$ but that the statements (i) "In any ring $A, J(A) \cap F(A)=0$ " and (ii) "Any radical ring (in the sense of Jacobson) which contains no proper nonzero ideal is a zero ring" are equivalent. Whether or not (ii) is true is an open question. Finally, if $A$ satisfies the descending chain condition for right ideals, $F(A)$ coincides with the largest regular ideal of $A$. (Received May 6, 1954.)

595t. T. A. Botts: On lattice embeddings for partially ordered sets.

A particularly simple suprema-preserving embedding of a partially ordered set in a complete distributive lattice is noted. Applied to the lattice of all additive topologies on a set $S$ [J. Wada, Lattices of spaces, Osaka Math. J. vol. 5 (1953) pp. 1-12], this yields a lattice of rather interesting elements, termed channel structures on $S$ [E. J. McShane, E. E. Floyd, and T. A. Botts, Channel spaces, to appear]. (Received April 29, 1954.)

\section{J. L. Brenner: An extension of Hadamard's condition that a matrix have nonzero determinant.}

If a square matrix $A=\left(a_{i j}\right)$ of complex numbers is such that the relations $\left|a_{k k}\right|$ $>\sum_{j \neq k}\left|a_{k j}\right|$ hold $(k=1,2, \cdots)$, its determinant is not 0 . This condition is generalized as follows. Let the rows of $A$ be partitioned into two or more sets, and suppose for each set of rows it is true that the absolute value of the principal minor exceeds the sum of the absolute values of the $-1+C_{r}^{n}$ nonprincipal minors on those rows. Then $\operatorname{det} A$ is not 0 . It is possible for $A$ to satisfy the general condition for one partitioning but not for a finer or coarser partitioning. Also, bounds for $\operatorname{det} A$ are found. The theorem gives, as usual, location theorems for the characteristic roots of an arbitrary square matrix. As Hadamard's original theorem has been generalized in the literature in various directions, so can this theorem be generalized in the parallel directions. (Received January 18, 1954.)

597. J. R. Byrne ( $p$ ) and R. A. Beaumont: Distributive polynomial functions and the construction of rings.

If $S_{1}, S_{2}, \cdots, S_{k}$ are subgroups of the additive group of a commutative ring $R$, then a distributive polynomial function $f$ from $S_{1} \times S_{2} \times \cdots \times S_{k}$ into $R$ is a function defined by a polynomial $f\left(x_{1}, x_{2}, \cdots, x_{k}\right)$ in $R\left[x_{1}, x_{2}, \cdots, x_{k}\right]$ which has the property that there exists an integer $m(1 \leqq m \leqq k)$ such that (1) $f\left(s_{1}+t_{1}, \cdots, s_{m}+t_{m}\right.$; $\left.s_{m+1}, \cdots, s_{k}\right)=f\left(s_{1}, \cdots, s_{m} ; s_{m+1}, \cdots, s_{k}\right)+f\left(t_{1}, \cdots, t_{m} ; s_{m+1}, \cdots, s_{k}\right)$ and (2) an analogous condition in the last $k-m$ arguments is satisfied, for all $s_{i}$ and $t_{i}$ in $S_{i}, i=1,2, \cdots, k$. The form of such a polynomial function is determined, and when $R$ has no proper divisors of zero, necessary and sufficient conditions that $f$ be distributive are found. These results are used in the construction of rings with additive group $S_{1} \oplus S_{2} \oplus \cdots \oplus S_{k}$, where $S_{1}, S_{2}, \cdots, S_{k}$ are ideals in $R$. (Received May 5, 1954.) 
598t. G. E. Forsythe: SWAC computes all 126 distinct semigroups of order 4.

By a "semigroup" is here meant a set of elements closed under an associative multiplication, with nothing else assumed. Two semigroups are "distinct" if they are neither isomorphic nor anti-isomorphic. Let $f(n)$ be the number of distinct semigroups of order $n$. It has been known that $f(1)=1, f(2)=4, f(3)=18$. The National Bureau of Standards Western Automatic Computer (SWAC) has now determined that $f(4)=126$. The author's code caused an exhaustive search through the $4^{16}$ possible multiplication tables. In about 100 minutes (reduced to about 80 minutes by $\mathrm{J}$. Selfridge) all 3492 semigroups of order 4 were punched out. SWAC later reduced these to an "alphabetized" list of normalized multiplication tables of the 126 distinct semigroups. Of these, 58 are commutative. A hectographed (unpublished?) list by K. S. Carman, J. C. Harden, and E. E. Posey of 121 distinct semigroups of order 4 was examined by SWAC and shown to be without error (though incomplete). (Received June 10, 1954.)

599. Burrowes Hunt: $A$ Farey-dissection of the plane and the corresponding simultaneous-approximation algorithm.

The rational points in the plane are given homogeneous coordinates $(x, y, z)$, relatively prime integers with $z$ the denominator in nonhomogeneous coordinates. The Farey-dissection of order $N$ of the unit square in the first quadrant, extended over the plane by translation, is a dissection into triangles with vertices at all rational points with $z \leqq N$. To the triangle $T$ with vertices $V_{i}=\left(x_{i}, y_{i}, z_{i}\right), z_{1} \geqq z_{2} \geqq z_{3}$, is assigned the matrix $T$ whose rows are $V_{1}, V_{2}, V_{3}$, in this order. In the dissection of order $z_{2}+z_{3}, T$ is split into two triangles, of matrices $A T$ and $B T$, by drawing the line from $V_{1}$ to the mediant $V_{2}+V_{3}$ of the opposite side. $A$ and $B$ are the matrices which take the rows of $T$ into $V_{2}+V_{3}, V_{1}, V_{3}$ or $V_{2}+V_{3}, V_{1}, V_{2}$, respectively. The entire dissection is determined by assigning matrices to the four triangles with bases the sides of the unit square and common "highest" vertex $(1,1,2)$, and using only the operations $A$ and $B . V_{3}$ is called an approximation to every point in or on $T$. The approximation to $P=(\xi, \eta, 1)$ is periodic if for some $T$, and $W$ some word in $A$ and $B, P$ is in $W^{n} T$ for $n=0,1,2, \ldots$. If periodic, then $\xi$ and $\eta$ are numbers in the field of a root of a cubic equation over $R$. The converse proposition is as yet neither proved nor disproved. (Received May 3, 1954.)

\section{D. H. Lehmer: Remarks on powers of a rational number.}

The distribution of the fractional parts of the successive integral powers of a rational number $r>1$ is not completely known. When $r=3 / 2$ this problem prevents the complete solution of Waring's Theorem. By using the SWAC it is shown that Waring's Theorem for $n$th powers is true for $n \leqq 5000$ because the fractional part of $(3 / 2)^{k}$ is sufficiently large for no value of $k \leqq 5000$. It is proved on the other hand that the fractional parts of $(1+1 / q)^{k}$, where $q$ is a fixed integer, are not randomly distributed. It is conjectured that this is the case for $(p / q)^{k}$. The conjecture has been verified for $q<p<17$. (Received March 15, 1954.)

601. Emma Lehmer: Period equations applied to residue difference sets.

It has been shown by Hall and Ryser that in a difference set of $k$ elements, modulo 
$p$, each difference occurring $\lambda$ times, all the prime divisors $q$ of $k-\lambda$ are multipliers provided $q>\lambda$. The last proviso, although essential to the proof, does not appear to be necessary in all cases. For residue difference sets the multipliers coincide with the residues. In this paper we show that all divisors of $k-\lambda$ are multipliers for all known residue difference sets. The theorem is obvious for quadratic residue sets. For quartic residue sets, discussed by Chowla, it is a special case of a theorem stated without proof by Sylvester to the effect that all divisors of $\left(3 p+x^{2}\right) / 16$ are quartic residues of $p=x^{2}+4 y^{2}, y$ odd. For octic difference sets, discussed by the author, this follows from a theorem, proved in the present paper, that all the divisors of $\left(7 p+a^{2}\right) / 64$ are octic residues of $p=a^{2}+2 b^{2}=9 a^{2}+4 y^{2}=16 n+9$. The proofs are based on a theorem of Kummer about the divisors of numbers represented by the cyclotomic period equations. The cubic period equation yields a similar result, namely that the factors of $\left(p+B^{2}\right) / 4$ are cubic residues of $p=4 A^{2}+27 B^{2}$. This can be applied with $B=1$ to mixed sextic residue difference sets of Marshall Hall, who gave a proof, in this special case, using the law of cubic reciprocity. (Received March 15, 1954.)

\section{E. A. Maier and Ivan Niven (p): On the Löwenheim-Müller verification theorem.}

The following generalization of the Löwenheim-Müller verification theorem (cf. L. Löwenheim, Über die Auflösung von Gleichungen im logischen Gebietekalkul, Math. Ann. vol. 68 (1910) p. 179) is proved. Write $a b, a+b, a^{\prime}$ and $b^{\prime}$ for the intersection, union, and complements of elements $a$ and $b$ of any Boolean algebra, and let 0 and 1 denote the null and universal elements. Let $f\left(x_{1}, \cdots, x_{n}\right)$ denote any polynomial in $n$ indeterminates $x_{1}, \cdots, x_{n}$ and their complements. Then any inclusion relation $f\left(x_{1}, \cdots, x_{n}\right) \subset g\left(x_{1}, \cdots, x_{n}\right)$ holds for all elements of the algebra if it holds for the $2^{n}$ cases where $x_{1}=0$ or $1, x_{2}=0$ or $1, \cdots, x_{n}=0$ or 1 . (Received May $3,1954$. )

\section{3t. D. H. Wagner: On free products of groups.}

The reduction procedure mentioned in the preliminary report (cf. Bull. Amer. Math. Soc. Abstract 58-1-10) is applied to prescribe a recursive procedure for deciding whether or not a given element of a free product belongs to the subgroup generated by a given finite subset. It is assumed that a procedure is at hand for deciding the corresponding question within each of the given free factors. (Received April 8, 1954.)

604t. N. A. Wiegmann: Some theorems on matrices with real quaternion elements.

Certain basic properties of real quaternion matrices have been developed in earlier papers (L. A. Wolf, Bull. Amer. Math. Soc. vol. 42 (1936) pp. 737-743; H. C. Lee, Proc. Royal Irish Academy vol. 52 (1949) pp. 253-260). Further properties of such matrices are developed here: After observing that an analogue of the Jordan normal form is implicit in the first reference, some properties of commutative quaternion matrices are obtained; it is shown that a polar representation is possible for quaternion matrices; certain properties of normal quaternion matrices are verified; if $A$ is a quaternion matrix, it is shown that there exist unitary quaternion matrices $U$ and $V$ such that $U A V$ is real and diagonal; and some results related to this latter transformation are obtained. (Received April 9, 1954.) 


\section{ANALYsis}

\section{5t. Richard Arens and A. P. Calderón: Analytic functions of several Banach algebra elements.}

Let $A$ be a commutative Banach algebra with unit, $M$ its space of maximal ideals, $a_{1}, \cdots, a_{n}$ a fixed set of elements of $A$. The set of all $\left(a_{1}(m), \cdots, a_{n}(m)\right)$ for $m$ in $M$ is the joint spectrum $S(A)$ (of $a_{1}, \cdots, a_{n}$ ) relative to $A$. Theorem 1: If $F$ is holomorphic in a neighborhood of $S(A)$, then there is an $a$ in $A$ such that $a(m)$ $=F\left(a_{1}(m), \cdots, a_{n}(m)\right)$ for all $m$. Theorem 2 extends this to (many-valued) analytic functions in a manner which for square roots comes down to this: if a continuous complex function $f$ can be found so that $0 \neq f(m)^{2}=a_{1}(m)$ for all $m$, then $f(m)=a(m)$ for some $a$ in $A$, i.e., $a_{1}$ has a square root mod the radical. Use is made of the following lemma. Let $\mathcal{A}$ be the system of finitely generated subalgebras of $A$ which contain $a_{1}, \cdots, a_{n}$, directed by anti-inclusion. Then $\lim _{B} \in \mathcal{C} S(B)=S(A)$. Thereby Theorem 1 is reduced to G. E. Silov's original theorem (Mat. Sbornik vol. 32 (1953) pp. 353364 ), which is like Theorem 1 except that $a_{1}, \cdots, a_{n}$ (and 1) are supposed to generate $A$. (Received May 6, 1954.)

\section{M. G. Arsove: Some conditions ensuring absolute continuity of the mass distribution for a potential.}

The conditions stated herein apply the upper and lower Blaschke operators $\bar{\Delta}^{B} u$ and $\Delta^{B} u$, defined respectively as the lim sup and $\lim$ inf of $\left(4 / r^{2}\right)\left[(1 / 2 \pi) \int_{0}^{2 \pi} u\left(z+r e^{i \theta}\right) d \theta\right.$ $-u(\bar{z})]$ as $r \rightarrow 0$. Theorem: Let $\Omega$ be an open set and $u$ a subharmonic function on $\Omega$ having the property that the set $\{z: u(z)=-\infty\}$ supports none of the mass of $u$; if $\bar{\Delta}^{B} u<+\infty$, except perhaps on a Borel $F_{\sigma}$ set of capacity zero, then the mass distribution for $u$ is absolutely continuous. Corollary: let $w$ be continuous on $\Omega$; if $\bar{\Delta}^{B} w$ and $\Delta^{B} w$ are finite, except perhaps on an $F_{\sigma}$ of capacity zero, and if $\Delta^{B} w$ is locally essentially bounded below, then $w$ can be represented as the difference of two continuous subharmonic functions having absolutely continuous mass distributions. These results, closely related to theorems of Rudin (Trans. Amer. Math. Soc. vol. 68 (1950) pp. 278-286), are proved by means of the Looman-Menchoff techniques (see the author's abstract in the Proceedings of the International Congress of Mathematicians, Amsterdam, 1954). (Received May 5, 1954.)

607t. M. M. Day: Strict convexity and smoothness. Preliminary report.

If $B$ is a normed linear space, say that $B$ is (a) $\mathrm{sc}$, (b) $\mathrm{sm}$, or (c) $\mathrm{scm}$ if $B$ is isomorphic to a normed space $B^{\prime}$ in which the unit sphere is (a) strictly convex, (b) smooth, or (c) both. Clarkson (Trans. Amer. Math. Soc. (1936)) showed that every separable space is sc, and Klee (Trans. Amer. Math. Soc. (1953)) showed that if $S$ is an arbitrary index set, then $l_{1}(S)$ is sc. It is proved here that: (1) Every separable $B$ is scm. (2) $c_{0}(S)$ is sc and sm, all $S$. (3) $l_{1}(S)$ is not sm if $S$ is uncountable. (4) $m_{0}(S)$, $=$ set of bounded real functions on $S$ which vanish outside some countable set, is not sc if $S$ is uncountable and is not sm if $S$ is infinite. (Received May 7, 1954.)

608t. J. B. Diaz and G. S. S. Ludford: On two methods of generating solutions of linear partial differential equations by means of definite integrals. 
(i) J. Le Roux (Ann. Ecole Norm. (1895)) proved, among other things, that if $U(x, y ; \alpha)$ is a solution, containing a parameter $\alpha$, of the linear equation $\left(^{*}\right) L(u)$ $\equiv u_{x y}+a(x, y) u_{x}+b(x, y) u_{y}+c(x, y) u=0$, then $\int_{\alpha_{0}}^{x} U(x, y ; \alpha) f(\alpha) d \alpha$ (with fixed limit $\left.\alpha_{0}\right)$ generates solutions of $\left({ }^{*}\right)$ from arbitrary functions $f(\alpha)$ provided $U(x, y ; \alpha)$ satisfies the characteristic condition $U_{y}+a(\alpha, y) U=0$ for $x=\alpha$. (ii) The kernel of S. Bergman's "integral operator" method is the theorem: If $E(x, y, t)$ is a solution of $\left(1-t^{2}\right)\left(E_{y t}+a E_{t}\right)-\left(E_{y}+a E\right) / t+2 x t L(E)=0$ such that $\left(1-t^{2}\right)^{1 / 2}\left(E_{y}+a E\right) / x t$ is continuous for $t=0$ and tends to zero for each $(x, y)$ as $t \rightarrow \pm 1$, then $\int_{-1}^{+1} E(x, y, t)$ - $f\left[x\left(1-t^{2}\right) / 2\right] d t /\left(1-t^{2}\right)^{1 / 2}$ generates solutions of $\left(^{*}\right)$ from arbitrary functions $f$ of a single variable. (iii) In this paper it is shown that the theorem in (ii) is equivalent to that in (i), and that in applications to the equations of plane compressible flow, the method of Le Roux yields simpler results (in particular, since in this case $a, b, c$ are functions of $x-y$ alone, one need only look for a single solution $\tilde{U}(x, y)$ of $\left({ }^{*}\right)$ and then write $U(x, y ; \alpha)=\widetilde{U}(x-\alpha, y-\alpha))$. In all these considerations the independent variables $x, y$ may be either real or complex. (Received April 12,1954.)

\section{9t. Jacob Feldman and R. V. Kadison: The uniform closure of the set of regular operators in a ring of operators.}

A precise description is given for those operators uniformly approximable by regular operators in a ring $R$. It is proved that $A$ is such an operator if and only if for each $\epsilon>0$ there exists a subspace $M$ belonging to $R$, containing the null space $N$ of $A$, such that $\|A M\|<\epsilon$ and $M$ and $H \Theta(A(H \Theta M))$ are equivalent (in the sense of Murray-von Neumann, On rings of operators, Ann. of Math. vol. 37 (1936) pp. 116-229). In the special case where $R$ is the ring of all bounded operators on a separable Hilbert space, this statement reduces to the following simple form: An operator $A$ is not a uniform limit of regular operators if and only if it is the product of a regular operator and a partial isometry between subspaces with unequal codimensions. (Received March 17, 1954.)

\section{R. D. James: Summable trigonometric series.}

The author has previously defined a $P^{n}$-integral of Perron type which goes directly to a generalized $n$th primitive (Trans. Amer. Math. Soc. vol. 76 (1954) pp. 149176). It is shown in the present paper that if a trigonometric series is summable $(C, k), k=0,1,2, \cdots$, to a function $f(x)$ for $0 \leqq x \leqq 2 \pi$, and satisfies certain other conditions, then $f(x)$ is necessarily $P^{k+2}$-integrable. Moreover, the coefficients of the trigonometric series are given by obvious modifications of the usual formulas for Fourier coefficients. (Received April 12, 1954.)

\section{1t. G. L. Krabbe: The Titchmarsh semi-group in Banach-space.}

Suppose $p>1$ and let $T_{\alpha}$ be the transformation defined as follows: if $\|a\|_{p}$ $=\left(\sum_{\nu=-\infty}^{\infty}\left|a_{\nu}\right|^{1 / 1 / p}<\infty\right.$, then $T_{\alpha} a$ is the sequence $c$ such that $c_{n}=\sum_{\nu=-\infty}^{\infty} a_{\nu}(-1)^{n+\nu}$ $\cdot \sin \alpha \pi /(n+\alpha-\nu) \pi(n=0, \pm 1, \pm 2, \cdots)$. Theorem I: There exists a nondecreasing function $f$ such that $\left\|T_{\alpha} a\right\|_{p} \leqq\|a\|_{p} f(|\operatorname{Im} \alpha|)$ when $\|a\|_{p}<\infty$. From this follows directly that $T_{\alpha}$ is an entire function of $\alpha$, and $T_{\alpha} T_{\lambda} a=T_{\alpha+\lambda} a, T_{0} a=a$ when $\|a\|_{p}<\infty$. E. Hille pointed out [Mathematical Reviews vol. 8 (1947) p. 659] that these results hold when $p=2$. A transformation $G$ (related to Hilbert's form) is defined as follows: if $\|a\|_{p}<\infty$, then $G a$ is the sequence $x$ such that $x_{n}=\sum_{\nu--\infty}^{\infty} a_{\nu}(-1)^{n+\nu}(n-\nu)^{-1}$, $\nu \neq n(n=0, \pm 1, \pm 2, \cdots)$. Among further immediate consequences of Theorem I is derived the fact that $G$ is a bounded operator; $G$ is the infinitesimal generator of 
the semi-group $\left\{T_{\alpha} ; \alpha\right\}$, and $T_{\alpha}=\exp \alpha G$ for all complex $\alpha$. The proof of Theorem I generalizes the method initiated by E. C. Titchmarsh in a special case [Math. Zeit. vol. 25 (1926) pp. 321-333]. (Received April 16, 1954.)

612t. A. J. Lohwater: The boundary values of pseudo-analytic functions.

Let $P$ denote the class of pseudo-analytic functions having bounded dilatation coefficient in $|z|<1$ for which $\lim _{r \rightarrow 1}\left|f\left(r e^{i \theta}\right)\right|=1$ for almost all $e^{i \theta}$ on $|z|=1$. It is shown that if $|f(z)|<1$, then every omitted value $\alpha$ with $|\alpha|<1$ is an asymptotic value. Extensions are made to nonbounded functions of $P$, and applications are made to the theory of the distribution of values of functions of $P$. (Received May 6,1954.)

\section{3t. J. S. MacNerney: Stieltjes integrals in linear spaces.}

Let $S$ be a linear normed complete space (norm $x=\|x\|$ ), and $B$ the LNC space of continuous linear transformations from $S$ into $S$ (norm $T=|T|=$ LUB $\|T x\|$ for $\|x\|$ $\leqq 1)$. Stieltjes-type integrals among functions from the real numbers into $S$ and into $B$ are considered; boundedness and bounded variation for functions from the real numbers to $S$ or to $B$ are defined in terms of the respective norms. H. S. Wall's theory of harmonic matrices [Bull. Amer. Math. Soc. Abstract 60-1-132] is extended to a theory of harmonic operators and associated integral equations. Application is also made to R. Nevanlinna's derivative concept [Math. Scand. vol. 1 (1953) pp. 104-112] : if $D$ is a locally convex, connected set in $S$ and $G$ is a function from $D$ to $B$ which is continuous in the norm topology of $B$ then, in order that there exist a function $f$ from $D$ to $S$ such that $f^{\prime}=G$ on $D$, it is necessary and sufficient that $\int_{0}^{1} G(z) \cdot d z=0$ for each continuous function $z$ from the number interval $[0,1]$ to $D$, of bounded variation on $[0,1]$, such that $z(0)=z(1)$. (Received April 29, 1954).

\section{4t. John Nash: $C^{3}$ isometric imbeddings.}

Given a closed $n$-manifold with $C^{3}$ metric one obtains a $C^{3}$ isometric imbedding in $E^{2 n^{2} / 2+3 n / 2}$ by a process of successive approximations. First one imbeds it in $n(n+1) / 2$ $+2 n$ dimensions in such a way as to be susceptible to the perturbation process. Then one employs $n(2 n+1)$ new dimensions to absorb almost all of the metric correction needed. The perturbation involves only the first $n(n+1) / 2+2 n$ imbedding functions. Each point of the manifold is moved in a direction orthogonal to the imbedding. This makes the metric effect depend on the second derivative matrix of the $n(n+1) / 2$ $+2 n$ functions. This gives rise to a system of linear equations in the perturbation vector components. A systematic global solution of these gives the perturbation as a function of the metric change desired. In connection with use of successive perturbations smoothing techniques must be applied to the functions involved in order to achieve convergence. The low frequency part of the correction must be made first. If the metric is $C^{k}, k \geqq 3$, one obtains a $C^{k}$ imbedding. Undoubtedly the dimensionality $2 n^{2} / 2+3 n / 2$ can be reduced and probably the process will extend to the $C^{2}$ and analytic cases. (Received June 8, 1954.)

615t. Vikramaditya Singh and W. J. Thron: On the number of singular points, located on the unit circle, of certain functions represented by $C$-fractions.

The following theorem has been proved: Let the continued fraction 1 $+K_{n=1}^{\infty}\left(d_{n} z^{\alpha_{n}} / 1\right)$, where $d_{n} \neq 0, \alpha_{n}$ a positive integer for all $n \geqq 1$, satisfy conditions 
(i) $\lim _{n \rightarrow \infty}\left(4\left|d_{n}\right|\right)^{1 / \alpha_{n}}=1$ and (ii) $\lim _{k \rightarrow \infty} \alpha_{n_{k}}=\infty$ where $\lim _{k \rightarrow \infty} n_{k} / k<1 / 2$. Introduce $\lim \inf _{n \rightarrow \infty} \rho_{n} /\left(h_{n}-\rho_{n}\right)=k$ where $h_{n}=\sum_{\nu=1}^{n+1} \alpha_{\nu}$ and $\rho_{n}$ is the maximum of the degrees of $A_{n}^{*}(z)$ and $B_{n}^{*}(z)$ where $A_{n}^{*}(z) / B_{n}^{*}(z)$ denotes the $n$th approximant of the continued fraction if the $d_{n}$ are replaced by their moduli. Then the function represented by the continued fraction has at least two singularities on the unit circle if $1 / 2 \leqq k<1$, and for $0<k<1 / 2$ the number of singularities on the unit circle is $\left[(\pi / 2) \cos ^{-1}(1 /(1+2 k))\right]$ +1 . Thus in particular, if $k=0$, the function has the unit circle as a natural boundary. This is an improvement of an earlier result by one of the authors [W. J. Thron, Duke Math. J. vol. 20 (1953) pp. 195-198]. (Received May 3, 1954.)

616t. D. A. Storvick: On meromorphic functions of bounded characteristic.

Let $f(z)$ be meromorphic with bounded characteristic in $|z|<1$ and let the radial limits of $f(z)$ have modulus 1 for almost all $z$ on $|z|=1$. Assume that $f(z)$ takes on values of modulus both greater than and less than 1. It is proved that, unless $f(z)$ is a schlicht function which maps $|z|<1$ onto the $w$-plane slit along an arc of $|w|=1$, every value of modulus 1 which is assumed only finitely often (or omitted) by $f(z)$ in $|z|<1$ is an asymptotic value of $f(z)$. This extends results of Hössjer [Acta. Univ. Szeged. vol. 5 (1930)] and Seidel [Trans. Amer. Math. Soc. vol. 36 (1934)], and is related to a result of Lehto [Ann. Acad. Sci. Fennicae (A) vol. 160 (1953)]. (Received April 22, 1954.)

\section{Bertram Yood: Periodic mappings on Banach algebras.}

Let $B$ be a semi-simple real Banach algebra. An automorphism or anti-automorphism $T$ of $B$ is called periodic with period $n$ if $n$ is the smallest positive integer such that $T^{n}=I$, the identity. It is shown that if $n=3$ or $2^{m}$ and if the set $\left\{x \in B \mid\left(I+T+\cdots+T^{n-1}\right)(x)=0\right\}$ is closed, then $T$ is continuous. Also if every $T$ of period two on every closed semi-simple sub-algebra of $B$ is continuous, then every $T$ of period $2^{m}$ on $B$ is continuous. (Received May 6,1954 .)

\section{ApPlied Mathematics}

\section{8t. Elvy L. Fredrickson: Applications of the Schmidt theory to} nonlinear integral equations.

The method of Erhard Schmidt [Math. Ann. vol. 65 (1908) pp. 370-430] for solving nonlinear integral equations is here applied to specific problems. Approximate solutions are obtained for: (1) Hamel's problem of the vibrating pendulum, (2) Duffing's equation, (3) an equation of Chandrasekhar which governs the angular distribution of radiation at the surface of a plane parallel stellar atmosphere. Work sponsored by U. S. Army Office of Ordnance Research under Contract DA-04-200ORD-177. (Received May 10, 1954.)

\section{9t. F. J. Murray: Mechanisms and robots.}

A mechanism is a device with $N$ states $\rho_{1}, \cdots, \rho_{N}$ which change automatically and this change $\rho_{i} \rightarrow \rho_{j}$ is given by a function $R i=j$. The behavior of a mechanism is specified by an iterative structure which is independent of the enumeration of the states. The number of such structures can be obtained using methods of G. P6lya (Acta Math. vol. 68 (1938) pp. 145-254). The relation with Boolean algebra is readily obtained. If the change of state is dependent on the environment, the mechanism is termed a robot. Robots which have specified probability responses to changes in the 
environment are described. A general method for constructing mechanisms and robots is indicated. Mathematical notions are used rather than the methods of formal logic and consequently much irrelevant discussion is avoided. (Received April 12, 1954.)

620t. L. B. Rall: Error bounds for iterative solutions of Fredholm integral equations.

The integral equation of Fredholm type and second kind is regarded as an operational equation $y=(I-\lambda K) x$ in the space $(C)$ of functions which are real and continuous on a closed interval $[a, b]$. A general iterative method $x_{m}=x_{m-1}-P(I-\lambda K) x_{m-1}$ $+P y, P$ an operator in $(C)$, converges for arbitrary $x_{0} \in(C)$ provided that $M(I-Q)<1$ or $Q$ is positive definite and $M(Q)<1$, where $Q=P(I-\lambda K)$, and $M(R)$ denotes the upper bound of the operator $R$ in $(C)$. Error estimates for the norm $\left\|x-x_{m}\right\|$ are obtained for the general process; this process is specialized to obtain the iterative methods due to C. Neumann, G. Wiarda, H. Buckner, P. Samuelson, and C. Wagner, and explicit error estimates for these methods. The general error bounds are extended to include the error introduced by the use of methods of approximate integration, and are illustrated by their application to a numerical example. [Work sponsored by U.S. Army, Office of Ordnance Research, Contract DA-04-200-ORD-177.] (Received May 10, 1954.)

\section{W. M. Stone (p) and Ray Lee: On the mean value Doppler} frequency spectrum of the return from a plane earth.

Consider a radar moving above a plane earth along a line in a plane vertical to the earth. The cone of radio energy, directed along a second arbitrarily oriented line, illuminates a conic section on the earth's surface. Under the assumption that each element of the illuminated area radiates a mean return signal in all directions with an appropriate Doppler shift in frequency, it is possible to integrate the resulting inverse fifth power function over the area of the conic section. The spectrum function is somewhat lengthy but is surprisingly elementary in form. Four independent parameters are involved, the three direction cosines associated with the line of motion and the central line of the cone of energy, plus the generating angle of the cone. Various properties involving the slope of the spectrum function, total power, etc., are obtained by elementary means. (Received May 4, 1954.)

\section{2t. Alexander Weinstein: A unified theory of the radiation prob- lem and of the Tricomi-Germain problem.}

Let (1) $L_{k} u=u_{x x}-u_{y y}-(k / y) u_{y}$. The solution $u=u^{k}(x, y)$ of $L_{k} u=0,-\infty<k<1$, which satisfies the conditions (2) $u^{k}(x, 0)=f(x)$ and (3) $u^{k}(x, y)=0$ for $x=y$ is given by the formula (4) $u^{k}(x, y)=\Gamma^{-1}(1-k)(\partial / \partial x)^{n+1} \int_{0}^{x-y} g(\xi)\left[(x-\xi)^{2}-y^{2}\right]^{-k / 2} d \xi$ where $g(\xi)$ denotes the Riemann-Liouville integral $I^{n+k} f(\xi)$. Here $-n$ denotes the greatest integer not exceeding $k ; n=0,1,2, \cdots$. In order to fulfill all conditions of the problem, $f(x)$ has to satisfy certain differentiability conditions and has to vanish together with a certain number of derivatives at $x=0$. This problem is for $k=-n$ a new formulation of the radiation problem for the wave equation in a space of $3+n$ dimensions. The cylindrical waves are obtained in the limiting case $k=1$. The new treatment furnishes a complete solution of a problem discussed in Courant-Hilbert, vol. 2, pp. 403-408, 415-416. For $k=1 / 3$ the above unified formula gives a new solution of the Tricomi problem in which the unknown function is prescribed on the parabolic line and vanishes on one characteristic. An explicit solution of this last problem was recently given 
by P. Germain and R. Bader (Rend. Circ. Mat. Palermo vol. 2 (1953) pp. 53-69, especially p. 63). Their solution, however, can be extended only to the range $0<k<1$. In this range both solutions coincide in view of a uniqueness theorem of $\mathrm{C}$. Morawetz (Bull. Amer. Math. Soc. vol. 59 (1953) p. 352). This research was supported by the Office of Scientific Research, U.S.A.F. (Received May 6, 1954.)

\section{GEOMETRY}

623. D. B. Dekker: Twisted curves and the mean-value proposition.

The mean-value proposition in the small states that between any two sufficiently close points of a curve there exists a third point of the curve at which the tangent is parallel to the chord joining the two points. The mean-value proposition in the large states that for each chord of a curve there exists a parallel tangent. Both of these propositions are obviously true for any plane differentiable curve. By use of Taylor formulas with remainders it is shown that any curve in $E_{3}$ belonging to class $C^{3}$ with nonvanishing curvature at each point is a plane curve if and only if the mean-value proposition in the small is true. Also it is shown that any curve in $E^{3}$ belonging to class $C^{1}$ with a nonvanishing tangent vector at each point and with a spherical indicatrix of tangents of dimension less than or equal to one is a plane curve if and only if the mean-value proposition in the large is true. The proof here involves a consideration of the dimension of the spherical indicatrix of chords. (Received May 3, 1954.)

\section{4t. V. L. Klee: Common secants for plane convex sets.}

A secant of a plane set $A$ is a line which intersects $A$. Theorem: Suppose $F$ is a family of connected plane sets, either $F$ is finite or each member of $F$ is compact, and there is a line in the plane none of whose parallels intersects more than one member of $F$. Then if each three members of $F$ have a common secant, there is a secant common to all members of $F$. (With "three" replaced by "four" this has been proved by Vincensini. With "connected plane sets" replaced by "mutually parallel line segments," it has been proved by Santalo and by Rademacher and Schoenberg.) The paper will appear in Proc. Amer. Math. Soc. (Received April 26, 1954.)

\section{LoGic and Foundations}

\section{5t. A. R. Schweitzer: Connecting links between Grassmann's space} analysis and integral equation theory.

Grassmann's space analysis is concerned with the concepts determinant (outer product), quadratic form (inner product), function space (Grassmann's concept of units). That a type of function space was known to Grassmann is clearly indicated by his phrase, "wenn Funktionen als Einheiten gesetzt werden" $\left(A_{2}\right.$, Abschnitt II, Kapitel 1, §5, Nr. 392). The preceding concepts have counterparts in integral equation theory. Transition from the former class of concepts to the latter involves passage from the finite to the infinite. Another link between Grassmann's theory and integral equation theory is the concept functional equation (Fréchet, Nouv. Ann. de Math. (4) vol. 9, p. 150). Reference is made to G. Kowalewski, Einfilhrung in die Determinantentheorie einschliesslich der Fredholmschen Determinanten (Berlin, 1942); G. Kowalewski, Ueber Funktionenräume (Sitzungsber. der kaiserl. Akad. der Wiss. in Wien. Mathem-naturw. Klasse; Bd. CXX Abt. Ila. Jänner 1911); D. Hilbert, Quadratische Formen: Grundzilge einer allgemeinen Theorie der linearen Integralgleichungen. Reference is also made to Encyk. der Mathem. Wiss., Bd. II, Teil 3, Heft 9 (p. 1434, note); Heft 7 (pp. 1025-1028). (Received April 23, 1954.) 


\title{
TOPOLOGY
}

\author{
626t. Professor S. T. Hu: The canonical spaces of associative \\ algebras.
}

For any given associative algebra $A$ over a commutative field $F$ with a unity element and for each topology $T$ in $F$, a topological space $X$ is constructed in this paper and is called the canonical space of the algebra $A$ over the commutative field $F$ with topology $T . X$ turns out to be a Hausdorff space provided $F$ is such under the topology $T$. If $A$ is the residue algebra of the polynomial ring $K\left[x_{1}, \cdots, x_{n}\right]$ modulo an ideal $I$, and if $T$ is the usual topology of the topological field $F=K$ of complex numbers, then it is proved that the canonical space $X$ is homeomorphic with the algebraic variety $V$ in the $n$-dimensional complex affine space $K^{n}$ which consists of the common zeros of all polynomials in the ideal $I$. This implies that the algebraic variety $V$ is determined up to a homeomorphism by the purely algebraic structure of the residue algebra $K\left[x_{1}, \cdots, x_{n}\right] / I$ and the topology of the field $K$. It also gives the algebraic mechanism of this determination. If $F$ is the field $R$ of all real numbers with the usual topology and if $A$ is the algebra $C(S, R)$ of all continuous real-valued functions defined on a Hewitt space $S$, then it turns out that the canonical space $X$ is homeomorphic with $S$. (Received May 3, 1954.)

\section{7t. V. L. Klee: Some topological properties of convex sets.}

This paper continues the study of topological properties of infinite-dimensional normed linear spaces and their convex subsets. In addition to results stated in earlier abstracts (Bull. Amer. Math. Soc. Abstracts 59-3-321, 59-4-435), the following is proved: (1) Suppose $E$ is an infinite-dimensional Banach space, $X$ is a compact subset of $E$, and $f$ is a homeomorphism of $X$ into $E$. Suppose that either $E$ is Hilbert space or $X$ is finite-dimensional. Then there is an isotopy $\eta$ of $E$ onto $E$ such that $\eta_{0}$ is the identity map on $E$ and $\eta_{1} \mid X=f$. By elaboration of a mapping-technique introduced by Keller, the following theorems are proved: (2) For each positive integer $n$, the Hilbert parallelotope is $n$-point homogeneous. (3) If $C$ is a locally compact closed convex subset of a normed linear space, then there are cardinal numbers $m$ and $n$ with $0 \leqq m \leqq \boldsymbol{N}_{0}$ and $0 \leqq n<\boldsymbol{N}_{0}$ such that $C$ is homeomorphic with either $\left.[0,1]^{m} \times\right] 0,1\left[{ }^{n}\right.$ or $[0,1]^{m} \times[0,1[$. (Received April 26, 1954.)

\section{8t. Deane Montgomery and Hans Samelson: $A$ theorem on fixed} points of involutions in $S^{3}$.

Let $T$ be an orientation preserving semi-linear homeomorphism of period two of $S^{3}$ onto itself; further let $T$ be different from the identity and have at least one fixed point. It follows from work of P. A. Smith that $F$, the set of all fixed points, is a simple closed curve but it is not known whether $F$ is necessarily unknotted. This paper shows that, at any rate, $F$ cannot belong to a certain class of knots including the ordinary cloverleaf. Let $C_{1}$ and $C_{2}$ be two disjoint simple closed polygons in $S^{3}$; $C_{1}$ is called a parallel knot of order 2 if there exists a polyhedral Möbius band with $C_{1}$ as edge and $C_{2}$ as a middle curve, that is, $C_{2}$ is a generator of the fundamental group of the band. The theorem proved is as follows: Suppose the fixed point curve $F$ of the semi-linear involution $T$ of $S^{3}$ is a parallel knot of order two of $C$. Then $\pi_{1}\left(S^{3}-C\right)$ is infinite cyclic and the linking number of $F$ and $C$ is \pm 1 . (The work of the second author was supported by the National Science Foundation.) (Received May 4, 1954.) 


\section{9t. F. P. Peterson: Hopf Theorem Mod $C$.}

Let $C$ be a "class" of abelian groups in the sense of Serre (Ann. of Math. vol. 58, pp. 258-294) satisfying also his axiom $\left(\mathrm{II}_{B}\right)$, namely, $A \in C$ implies $A \otimes B \in C$ and Tor $(A, B) \in C$. The following dual (concerning cohomotopy and cohomology) to the Hurewicz theorem is proved. Let $(X, A)$ be a compact pair with dimension $X=N$. If $H^{i}(X, A) \in C$ for $i>n(n>(N+1) / 2)$, then $\pi^{i}(X, A) \in C$ for $i>n$ and $\pi^{n}(X, A) \rightarrow H^{n}(X, A)$ is a $C$-isomorphism. Using facts about $\pi_{i}\left(S^{n}\right)$, the conclusion of the above can be strengthened. Let $\alpha_{C}$ be the largest number such that $\pi_{i+j}\left(S^{i}\right) \in C$ for $0<j<\alpha_{C}$ (within the stable range of $\pi_{i+j}\left(S^{i}\right)$ ). The conclusion then reads that $\pi^{i}(X, A) \rightarrow H^{i}(X, A)$ is a $C$-isomorphism for $i>\operatorname{Max}\left((N+1) / 2, n-\alpha_{C}\right)$. With this relative theorem, it is easy to prove the following dual to the Whitehead isomorphism theorem. Let $X, Y$ be compact spaces of dimensions $M, N$ respectively. Let $f: X \rightarrow Y$ be a map, and let $n>\operatorname{Max}((M+2) / 2,(N+1) / 2)$. Then $f^{*}: H^{i}(Y) \rightarrow H^{i}(X)$ is a $C$-isomorphism for $i>n$ and $C$-onto for $i \geqq n$ if and only if $f^{\#}: \pi^{i}(Y) \rightarrow \pi^{i}(X)$ is a $C$-isomorphism for $i>n$ and $C$-onto for $i \geqq n$. (Received May 3, 1954.)

\section{0t. A. D. Wallace: Partial order and indecomposability.}

Let $X$ be a space and let $R$ be a closed subset of $X \times X$. For our present purpose it is convenient to write $x \leqq y$ if $(x, y) \in R$ and to assume that $\leqq$ is reflexive and transitive. It is natural to ask how the structure of $R$ is affected by the structure of $X$. In a note to appear in the Proceedings of this Society we prove the following: (i) For each $a \in X$ let $\{x \mid x \leqq a\}$ be connected; (ii) For each $x, y \in X$ let there exist $z \in X$ such that $z \leqq x$ and $z \leqq y$; (iii) Let $X$ be an indecomposable continuum-then $R=X \times X$. (Received April 5, 1954.)

\section{1t. A. D. Wallace: Two separation theorems.}

Theorem 1. Let $\mathcal{T}$ be a family of open sets in the space $X$ which satisfies: (i) If $T_{1}, T_{2} \in \mathcal{V}$, then $T_{1} \cup T_{2} \in \mathcal{V}$; (ii) If $U$ is open and if $F(U) \subset F(T)$ and if $T \in \mathcal{V}$, then $U \in \mathcal{T}$. Let $A$ and $B$ be subsets of $X$ such that $F(A)$ and $F(B)$ are compact and such that the interior of $A$ does not meet $B$ and vice versa. Suppose that, if $a \in F(A)$ and if $b \in F(B), a \in T$ and $b \in X \backslash T^{*}$ for some $T \in \mathcal{V}$. Then $A^{*} \subset T$ and $B^{*} \subset X \backslash T^{*}$ for some $T \in \mathcal{T}$. Theorem 2. Let $\mathcal{A}$ be a family of subsets of the space $X$ which satisfies: (i) If $A_{1}, A_{2} \in \mathcal{C}$, then $A_{1} \cup A_{2} \in \mathcal{C}$; (ii) if $U$ is open and if $F(U) \subset A \in \mathcal{C}$, then $U \cup A \in \mathcal{A}$. If $p \in X$ let $x \in C(p)$ if for no $A \in \mathcal{A} A$, does one have $x \in A^{0}$ and $p \in X \backslash A *$. Let $X$ be a rim compact Hausdorff. Then $C(p)$ is closed and if $F(C(p))$ is compact it follows that $C(p)$ is connected. To prove Theorem 2, a corollary of Theorem 1 is used. Let $X$ be $\operatorname{rim}$ compact Hausdorff, let $A$ be closed with $F(A)$ compact, and let $U$ be an open set about $A$. Then there is an open set $V$ about $A$ with $F(V)$ compact and with $V^{*} \subset U$. The above theorems are novel only in their generality and are useful only as a unifying device. (Received April 5, 1954.)

\section{2t. L. E. Ward, Jr.: A note on dendrites and trees.}

A partial order, $\leqq$, on a space $X$ is said to be semicontinuous if $L(x)=\{a: a \leqq x\}$ and $M(x)=\{a: x \leqq a\}$ are closed sets, for each $x \in X$. We shall say that $X$ is a tree if $X$ is a compact connected Hausdorff space in which every pair of distinct points is separated by a third point. Thus a dendrite is simply a metric tree. Theorem: Let $X$ be a compactum; then a necessary and sufficient condition that $X$ be a dendrite is that $X$ admit a partial order, $\leqq$, satisfying (i) $\leqq$ is semicontinuous, (ii) $\leqq$ is order 
dense, (iii) $L(x) \cap L(y)$ is a non-null chain for each $x, y \in X$, (iv) $M(x)-x$ is an open set for each $x \in X$. If the words "compact Hausdorff space" and "tree" are substituted for "compactum" and "dendrite," the theorem remains valid. (Received April 20, 1954.)

\title{
633t. G. W. Whitehead: Homotopy groups of joins and the groups
} $A_{q}(\pi ; G)$.

Let $X, Y$ be spaces, $X * Y$ their join. Suppose that $X$ is $(m-1)$-connected, $Y(n-1)$-connected. Then there is a spectral sequence $\left\{E_{r}\right\}$ such that $E_{2}^{p, a}$ $=H_{n+p}\left(Y ; \pi_{m+q}(X)\right)$ for $q \leqq m-2$ and $E_{\infty}$ is the graded group of $\sum_{n} \pi_{n}(X * Y)$ with respect to a suitable filtration. In particular, if $X=K(\pi, n), Y=K(G, n)$, then $\pi_{2 n+s+1}(X * Y) \approx H_{n+8}(\pi, n ; G)$ for $s \leqq m-3$. This gives a simple proof of the unpublished theorem of $\mathrm{H}$. Cartan that the stable Eilenberg-MacLane groups satisfy the condition $A_{q}(\pi ; G) \approx A_{q}(G ; \pi)$. (Received April 23, 1954.)

634t. G. W. Whitehead: On the homology suspension.

Let $B$ be an $n$-connected space, $F$ its space of loops, $A$ an abelian group. Then for $q \leqq 3 n-1$ there is an exact sequence $H_{q-1}(F ; A) \rightarrow^{\sigma} H_{q}(B ; A) \rightarrow^{\top} G_{q} \rightarrow^{\rho} H_{q-2}(F ; A)$ $\rightarrow{ }^{\sigma} H_{q-1}(B ; A)$, where $G_{q} \approx H_{q}(B \times B, B \vee B ; A) \approx H_{q-2}(F \times F, F \vee F ; A), \sigma$ is the suspension, $\tau: H_{q}(B ; A) \rightarrow H_{q}(B \times B, B \vee B ; A)$ is induced by the diagonal map, and $\rho: H_{q-2}(F \times F, F \vee F ; A) \rightarrow H_{q-2}(F ; A)$ is naturally related to the operation of path multiplication in $F$. A dual result holds for cohomology. If $\theta: H^{n}(K ; \pi) \rightarrow H^{q}(K ; A)$ is a universally defined cohomology operation, $u$ the element of $H^{q}(\pi, n ; A)$ which corresponds to $\theta$, and if $q \leqq 3 n-1$, then $\theta$ is additive if and only if $u$ is the suspension of an element of $H^{q^{+1}}(\pi, n+1 ; A)$. The latter result has been conjectured by Eilenberg and MacLane to hold for all $q$. (Received April 23, 1954.)

\author{
J. W. Green, \\ Associate Secretary
}

